\title{
Application of Non-Orthogonal Multiple Access in Cooperative Spectrum-Sharing Networks over Nakagami- $m$ Fading Channels
}

\author{
Lu Lv, Qiang Ni, Senior Member, IEEE, Zhiguo Ding, Senior Member, IEEE, and Jian Chen, Member, IEEE
}

\begin{abstract}
This paper proposes a novel non-orthogonal multiple access (NOMA)-based cooperative transmission scheme for a spectrum-sharing cognitive radio network, whereby a secondary transmitter (ST) serves as a relay and helps transmit the primary and secondary messages simultaneously with employing NOMA signaling. This cooperation is particularly useful when the ST has good channel conditions to a primary receiver but lacks of the radio spectrum. To evaluate the performance of the proposed scheme, the outage probability and system throughput for the primary and secondary networks are derived in closed forms. Simulation results demonstrate the superior performance gains for both networks thanks to the use of the proposed NOMAbased cooperative transmission scheme. It is also revealed that NOMA outperforms conventional orthogonal multiple access and achieves better spectrum utilization.
\end{abstract}

Index Terms-Non-orthogonal multiple access (NOMA), cooperative spectrum-sharing, Nakagami- $m$ fading.

\section{INTRODUCTION}

Non-orthogonal multiple access (NOMA) has been identified as a promising multiple access technique for the fifth generation $(5 \mathrm{G})$ mobile networks due to its superior spectral efficiency [1]-[3]. The rational is that multiple users' signals are superimposed at the transmitter with different power allocation coefficients, and the user with better channel conditions decodes its own signal by employing successive interference cancellation (SIC). In [4], a cooperative NOMA scheme has been proposed based on user cooperation. Using a dedicated relay, the performance of cooperative relaying networks via NOMA has been analyzed in [5].

Cognitive radio networks (CRNs) have been a new paradigm to enable more efficient use of the radio spectrum [6], [7]. By introducing NOMA to CRN, the higher spectral efficiency can be potentially achieved. However, little research has combined NOMA with CRN in an effective way. Recently, the outage performance of NOMA in underlay CRNs has been characterized in [8] with applying stochastic geometry tools. A cooperative NOMA scheme for reliability enhancement in cognitive multicast networks has been proposed in [9].

In this paper, we investigate the application of NOMA to a cooperative spectrum-sharing CRN. Unlike the underlay CRN which sets interference limit to the primary network [8], we consider a cooperative scenario where the primary

This work was supported in part by the EU FP7 CROWN project under Grant PIRSES-GA-2013-610524, by the National Natural Science Foundation of China under Grants 61540046 and 61601347 , and by the "111" project of China under Grant B38038. The associate editor coordinating the review of this manuscript and approving it for publication was Dr. M. Elkashlan.

L. Lv and J. Chen are with the State Key Laboratory of Integrated Service Networks, Xidian University, Xi'an 710071, China (e-mail: lulv@ @stu.xidian.edu.cn; jianchen@mail.xidian.edu.cn).

Q. $\mathrm{Ni}$ and $\mathrm{Z}$. Ding are with the School of Computing and Communications, Lancaster University, Lancaster LA1 4WA, U.K. (e-mail: q.ni@lancaster.ac.uk; z.ding@lancaster.ac.uk). and secondary networks actively seek opportunities to collaborate with each other. Compared with our prior work in [9], where the cooperative NOMA scheme slightly degrades the performance of the primary network, this paper proposes a win-win strategy. In particular, the secondary transmitter (ST) helps relay the information from the primary transmitter (PT) to the primary receiver (PR), meanwhile the ST utilizes the primary spectrum to serve its own secondary receiver (SR) for the compensation. This creates an incentive for the PT and ST to work together, and the ST is no longer constrained by low transmit power. The main contributions of this paper are three fold: First, we propose a novel NOMA-based cooperative transmission scheme to achieve better utilization of the spectrum, whereby the ST which has good channel conditions to the PR is recruited as a relay to assist the primary transmission, and in turn it can use the primary spectrum. To improve the spectral efficiency, the ST will adopt NOMA for the simultaneous primary and secondary transmissions. Consequently, an enhanced win-win situation can be achieved. Second, we derive closed-form expressions of the outage probability and the system throughput for both primary and secondary networks. To obtain further insights, asymptotic expressions for the outage probability are derived to validate the diversity order by the proposed scheme. Finally, numerical results are presented to demonstrate the effectiveness of the proposed scheme and the accuracy of the analytical results. Compared with conventional orthogonal multiple access (OMA)-based schemes, the performance in terms of the outage probability and the system throughput can be significantly improved.

\section{SySTEM MODEL}

Consider a cooperative spectrum-sharing network consisting of the primary and secondary nodes, denoted by PT, PR, $\mathrm{ST}$, and $\mathrm{SR}_{i}, i \in \mathcal{K}=\{1,2, \cdots, K\}$, respectively. Each node is equipped with a single antenna and operates in a half-duplex mode. All channels are assumed to experience independent and identically distributed (i.i.d.) Nakagami- $m$ fading. In particular, the channel gains from PT and ST to $\mathrm{SR}_{i}$ are denoted by $h_{p, i}$ and $h_{s, i}$. We have $E\left[\left|h_{p, i}\right|^{2}\right]=\Omega_{p, s}$ and $E\left[\left|h_{s, i}\right|^{2}\right]=\Omega_{s, s}$, whose fading parameter is $m$. The channel gains from PT to PR and ST are denoted by $f_{0}$ and $f_{1}$, and the channel gain from ST to PR is denoted by $f_{2}$. Similarly, we have $E\left[\left|f_{0}\right|^{2}\right]=\Omega_{f, 0}, E\left[\left|f_{1}\right|^{2}\right]=\Omega_{f, 1}$, and $E\left[\left|f_{2}\right|^{2}\right]=\Omega_{f, 2}$, with fading parameter $m$. In this paper, we assume all channels follow quasi-static fading, i.e. the channel gains remain to be constant within each transmission block but vary independently between different blocks. Without loss of generality, it is assumed that the users' channel gains in the secondary network are ordered as $\left|h_{s, 1}\right|^{2} \leq \cdots \leq\left|h_{s, K}\right|^{2}$. Let $x_{0}$ and $x_{i}, i \in \mathcal{K}$, denote the primary and secondary users' signals, with $E\left[x_{0}\right]=E\left[x_{i}\right]=0$ and $E\left[\left|x_{0}\right|^{2}\right]=E\left[\left|x_{i}\right|^{2}\right]=1$. 
The transmit power at $\mathrm{PT}$ and $\mathrm{ST}$ are denoted by $P_{p}$ and $P_{s}$, and the background noise is modeled by a zero-mean, complex Gaussian variable with variance $\sigma^{2}$.

As shown in Fig. 1 (a), the proposed cooperative NOMAbased transmission scheme consists of two phases. During the first transmission phase, $x_{0}$ is transmitted by PT, the received signal at PR is given by

$$
y_{p 1}=\sqrt{P_{p}} f_{0} x_{0}+n_{p 1}
$$

and the received signal at ST is expressed as

$$
y_{s}=\sqrt{P_{p}} f_{0} x_{0}+n_{s}
$$

where $n_{p 1}$ and $n_{s}$ are the noises during the first transmission phase. The condition that ST is able to successfully decode $x_{0}$ is: $\rho_{p}\left|f_{1}\right|^{2} \geq \psi_{0}$, where $\psi_{0}=2^{2 R_{0}}-1$ is the threshold for data decoding, $\bar{R}_{0}$ is the target data rate of PR, and $\rho_{p}=\frac{P_{p}}{\sigma^{2}}$ is the transmit signal-to-noise ratio (SNR) at PT. At the end of this phase, ST tries to decode $x_{0}$. If the decoding is successful, ST can regenerate $x_{0}$ and combine it with $x_{i}, i \in \mathcal{K}$, for relaying in the next NOMA transmission phase.

The received signal at $\mathrm{SR}_{k}$ is written as

$$
y_{s, k 1}=\sqrt{P_{p}} h_{s, k} x_{0}+n_{k 1}
$$

where $n_{k 1}$ is the noise during the first transmission phase.

During the second transmission phase, conditioned on ST succeeding in decoding, ST transmits the superimposed signals $\sum_{i=0}^{K} \sqrt{\alpha_{i} P_{s}} x_{i}$ to $\mathrm{PR}$ and $\mathrm{SR}_{i}$, where $x_{i}$ contains the information required by $\mathrm{SR}_{i}$ for $i \in \mathcal{K}$ or PR for $i=0$, and $\alpha_{i}$ denotes the power allocation coefficient of $x_{i}, i=0,1, \cdots, K$. Due to $\left|h_{s, 1}\right|^{2} \leq \cdots \leq\left|h_{s, K}\right|^{2}$ and the high priority of PR, we let $\alpha_{0} \geq \alpha_{1} \geq \cdots \geq \alpha_{K}$ with $\sum_{i=0}^{K} \alpha_{i}=1$ [8]. The received signals at $\mathrm{PR}$ during the second transmission phase are

$$
y_{p 2}=f_{2} \sum_{i=0}^{K} \sqrt{\alpha_{i} P_{s}} x_{i}+n_{p 2}
$$

where $n_{p 2}$ is the noise during the second transmission phase. On maximal-ratio combining (MRC), the achievable data rate at PR can be expressed as

$$
R_{p, 1}=\frac{1}{2} \log _{2}\left(1+\rho_{p}\left|f_{0}\right|^{2}+\frac{\rho_{s}\left|f_{2}\right|^{2} \alpha_{0}}{\rho_{s}\left|f_{2}\right|^{2} \sum_{i=1}^{K} \alpha_{i}+1}\right)
$$

where $\rho_{s}=\frac{P_{s}}{\sigma^{2}}$ is the transmit SNR at ST.

The observations at $\mathrm{SR}_{k}$ can be expressed as

$$
y_{s, k 2}=h_{s, k} \sum_{i=0}^{K} \sqrt{\alpha_{i} P_{s}} x_{i}+n_{k 2}
$$

where $n_{k 2}$ denotes the noise at $\mathrm{SR}_{k}$ during the second transmission phase. SIC will be carried out at $\mathrm{SR}_{k}$ to separate the multiplexed signals in the power domain. Specifically, $\mathbf{S R}_{k}$ will first detect $\mathrm{SR}_{j}$ 's signal, $j<k$, and then remove the signal from its observation in a successive manner [4]. While $\mathrm{SR}_{j}$ 's signal, $j>k$, will be treated as noise at $\mathrm{SR}_{k}$. Recalling that $x_{0}$ has been received in the first transmission phase, then $\mathrm{SR}_{k}$ will use MRC to detect $x_{0}$ in the SIC process. Thus, the achievable data rate, i.e. $j=0$, is expressed as

$$
R_{0 \rightarrow k}=\frac{1}{2} \log _{2}\left(1+\rho_{p}\left|h_{p, k}\right|^{2}+\frac{\rho_{s}\left|h_{s, k}\right|^{2} \alpha_{0}}{\rho_{s}\left|h_{s, k}\right|^{2} \sum_{i=1}^{K} \alpha_{i}+1}\right) .
$$

The achievable data rate for $\mathrm{SR}_{k}$ to detect $\mathrm{SR}_{j}$ 's signal, $1 \leq$ $j \leq k$, is given by

$$
R_{j \rightarrow k}=\frac{1}{2} \log _{2}\left(1+\frac{\rho_{s}\left|h_{s, k}\right|^{2} \alpha_{j}}{\rho_{s}\left|h_{s, k}\right|^{2} \sum_{i=j+1}^{K} \alpha_{i}+1}\right) .
$$

If $R_{j \rightarrow k} \geq R_{j}(j=0, \cdots, K)$, where $R_{j}$ is the target data rate of $x_{j}$, then $x_{j}$ can be decoded and removed by $\mathrm{SR}_{k}$. This process will terminate until $x_{k}$ can be successfully decoded at $\mathrm{SR}_{k}$. The achievable data rate at $\mathrm{SR}_{k}, k=1, \ldots, K-1$, is

$$
R_{k, 1}=\frac{1}{2} \log _{2}\left(1+\frac{\rho_{s}\left|h_{s, k}\right|^{2} \alpha_{k}}{\rho_{s}\left|h_{s, k}\right|^{2} \sum_{i=k+1}^{K} \alpha_{i}+1}\right) .
$$

Furthermore, the achievable data rate at $\mathrm{SR}_{K}$ can be obtained by $R_{K, 1}=\frac{1}{2} \log _{2}\left(1+\rho_{s}\left|h_{s, K}\right|^{2} \alpha_{K}\right)$.

On the other hand, when ST fails to decode $x_{0}$, it will remain silent in the second transmission phase. In this case, PR receives $x_{0}$ only from the first transmission phase. Thus, the achievable data rates at $\mathrm{PR}$ and $\mathrm{SR}_{k}$ are given by

$$
R_{p, 2}=\frac{1}{2} \log _{2}\left(1+\rho_{p}\left|f_{0}\right|^{2}\right), \quad R_{k, 2}=0 .
$$

\section{Performance AnAlysis}

This section provides a comprehensive performance analysis of primary and secondary networks achieved by the proposed NOMA-based cooperative transmission scheme.

\section{A. Primary Network}

1) Outage Probability: The outage probability of the primary network with the proposed NOMA-based cooperative transmission scheme can be formulated as

$$
P_{p}=\underbrace{\operatorname{Pr}\left(\left|f_{1}\right|^{2}>\frac{\psi_{0}}{\rho_{p}}, R_{p, 1}<R_{0}\right)}_{I_{1}}+\underbrace{\operatorname{Pr}\left(\left|f_{1}\right|^{2}<\frac{\psi_{0}}{\rho_{p}}, R_{p, 2}<R_{0}\right)}_{I_{2}} .
$$

Particularly, $I_{1}$ is for the event that ST can decode the primary user's signal correctly but the achievable rate at PR is smaller than the target data rate; and $I_{2}$ is for the event that neither ST or PR can decode the primary user's signal.

The following theorem provides the outage probability for the primary network with our proposed scheme.

Theorem 1: The outage probability of the primary network with the proposed scheme can be approximated as (12), shown at the top of the next page, where $N$ is a complexity-accuracy tradeoff parameter, $\varpi=\min \left(\psi_{0}, \frac{\alpha_{0}}{\sum_{i=1}^{K} \alpha_{i}}\right), \theta_{n}=\cos \left(\frac{2 n-1}{2 N} \pi\right)$, $b_{n}=\frac{\left(\theta_{n}+1\right) \varpi}{2}$, and $f_{Y}(y)$ is defined in Appendix A.

Proof: See Appendix A.

2) Asymptotic Analysis: We define the system $\mathrm{SNR}$ as $\bar{\rho}=$ $\rho_{p}=\mu \rho_{s}$ with $\mu=\rho_{p} / \rho_{s}$. With the help of $e^{x} \simeq 1-x$ for a sufficiently large system SNR, i.e. $\bar{\rho} \rightarrow \infty$, the asymptotic expression for (12) is given by

$$
P_{p} \stackrel{\bar{\rho} \rightarrow \infty}{\simeq} \frac{1}{\bar{\rho}^{2 m}}\left(\frac{\varpi \pi}{2 N} \sum_{n=1}^{N} c_{n} d_{n}+\chi_{m}\right)
$$

where $\chi_{m}=\frac{\left(m \psi_{0}\right)^{2 m}}{\left(\Omega_{f, 0} \Omega_{f, 1}\right)^{m}(m !)^{2}}, c_{n}=\sqrt{1-\theta_{n}^{2}}\left(\frac{\psi_{0}-b_{n}}{\Omega_{f, 0}}\right)^{m} / m$ !, and $d_{n}=\frac{m^{m}\left(b_{n} /\left(\alpha_{0}-\sum_{i=1}^{K} \alpha_{i}\right)\right)^{m-1}}{\left(\mu \Omega_{f, 2}\right)^{m} \Gamma(m)}$. Therefore, it is clear from (13) that a diversity order of $2 m$ is achieved for the primary network, thanks to the cooperative NOMA transmission.

3) Outage Throughput: In this paper, the transmitter sends information at a fixed rate and the throughput is determined by evaluating the outage probability. This implies that the receiver has to decode the signal block by block and thus it is important to study the outage throughput for practical implementations, which is given by

$$
\tau_{p}=\left(1-P_{p}\right) R_{0} .
$$




$$
\begin{aligned}
P_{p} \approx F_{Y}(\varpi) & -\frac{\varpi \pi}{2 N} \sum_{n=1}^{N} \sum_{s=0}^{m-1} \sum_{r=0}^{m-1} \sqrt{1-\theta_{n}^{2}} \exp \left(-\frac{m \psi_{0}}{\rho_{p} \Omega_{f, 1}}\right) \exp \left(\frac{m\left(b_{n}-\psi_{0}\right)}{\rho_{p} \Omega_{f, 0}}\right) \frac{\left(m \psi_{0} / \rho_{p} \Omega_{f, 1}\right)^{s}}{s !} \frac{\left(m\left(\psi_{0}-b_{n}\right)\right)^{r}}{r !\left(\rho_{p} \Omega_{f, 0}\right)^{r}} f_{Y}\left(b_{n}\right) \\
& +\left(1-\exp \left(-\frac{m \psi_{0}}{\rho_{p} \Omega_{f, 1}}\right) \sum_{s=0}^{m-1} \frac{\left(m \psi_{0} / \rho_{p} \Omega_{f, 1}\right)^{s}}{s !}\right) \exp \left(-\frac{m \psi_{0}}{\rho_{p} \Omega_{f, 0}}\right) \sum_{l=0}^{m-1} \frac{\left(m \psi_{0} / \rho_{p} \Omega_{f, 0}\right)^{l}}{l !}
\end{aligned}
$$

\section{B. Secondary Network}

1) Outage Probability: The outage event for $\mathrm{SR}_{k}$ is defined as follows. First, we denote $\mathcal{O}_{k, j} \triangleq\left\{R_{j \rightarrow k}<R_{j}\right\}$ as the event that $\mathrm{SR}_{k}$ cannot detect $\mathrm{SR}_{j}$ 's signal, $0 \leq j \leq k$, and $\mathcal{O}_{k, j}^{c}$ as the complementary set of $\mathcal{O}_{k, j}$. When $\mathrm{SR}_{k}$ decodes $x_{0}$ successfully, the outage probability can be expressed as

$$
\tilde{P}_{k}=1-\operatorname{Pr}\left(\mathcal{O}_{k, 0}^{c} \cap \cdots \cap \mathcal{O}_{k, k}^{c}\right) .
$$

Since $\mathrm{SR}_{k}$ will first adopt MRC to detect the primary user's signal (i.e. $j=0$ ), the event $\mathcal{O}_{k, 0}^{c}$ is given by

$$
\mathcal{O}_{k, 0}^{c} \triangleq\left\{\rho_{p}\left|h_{p, k}\right|^{2}+\frac{\rho_{s}\left|h_{s, k}\right|^{2} \alpha_{0}}{\rho_{s}\left|h_{s, k}\right|^{2} \sum_{i=1}^{K} \alpha_{i}+1}>\psi_{0}\right\}
$$

and the event $\mathcal{O}_{k, j}^{c}, 1 \leq j \leq k$, is formulated as

$$
\mathcal{O}_{k, j}^{c} \triangleq\left\{\frac{\rho_{s}\left|h_{s, k}\right|^{2} \alpha_{j}}{\rho_{s}\left|h_{s, k}\right|^{2} \sum_{i=j+1}^{K} \alpha_{i}+1}>\psi_{j}\right\}
$$

where $\psi_{j}=2^{2 R_{j}}-1, j \in \mathcal{K}$. In addition, note that the event $\mathcal{O}_{K, K}^{c}$ can be expressed as $\mathcal{O}_{K, K}^{c} \triangleq\left\{\rho_{s}\left|h_{s, K}\right|^{2}>\psi_{K}\right\}$.

Theorem 2: $\tilde{P}_{k}$ can be approximated as (18), shown at the top of the next page, where $N$ is a complexity-accuracy tradeoff parameter, $\phi_{n}=\cos \left(\frac{2 n-1}{2 N} \pi\right), \tau_{k}=\frac{K !}{(K-k) !(k-1) !}, \epsilon\left(\psi_{k}^{*}\right)=$ $\frac{\psi_{0}}{\rho_{p}}-\frac{\alpha_{0} \psi_{k}^{*}}{\rho_{p}\left(1+\psi_{k}^{*} \sum_{i=1}^{K} \alpha_{i}\right)}, \psi_{0}^{\prime}=\frac{\psi_{0}-\rho_{p}\left(\phi_{n}+1\right) \epsilon\left(\psi_{k}^{*}\right) / 2}{\alpha_{0}-\left(\psi_{0}-\rho_{p}\left(\phi_{n}+1\right) \epsilon\left(\psi_{k}^{*}\right) / 2\right) \sum_{i=1}^{K} \alpha_{i}}$, $f_{\left|h_{p, k}\right|^{2}}(z)$ and $\psi_{k}^{*}$ are defined in Appendix B.

Proof: See Appendix B.

The outage probability of $\mathrm{SR}_{k}$ with the proposed NOMAbased cooperative transmission scheme is given by

$$
P_{k}=\operatorname{Pr}\left(\rho_{p}\left|f_{1}\right|^{2}>\psi_{0}\right) \tilde{P}_{\text {out }, k}+\operatorname{Pr}\left(\rho_{p}\left|f_{1}\right|^{2}<\psi_{0}\right) .
$$

Substituting (18) and (28) into (19), with the help of Theorem 2, a closed-form expression for $P_{k}$ can be readily obtained.

2) Asymptotic Analysis: Using the same rationale with (13), an asymptotic expression for (19) can be expressed as

$$
P_{k} \stackrel{\rho_{s} \rightarrow \infty}{\simeq^{\infty}} \frac{1}{\rho_{s}^{m k}}\left(\tau_{k}^{\prime} \zeta_{m}+\frac{\epsilon\left(\psi_{k}^{*}\right) \pi}{2 N} \sum_{n=1}^{N} e_{n} \delta_{m}\right)
$$

where $\tau_{k}^{\prime}=\frac{K !}{(K-k) ! k !}, \zeta_{m}=\left(\frac{m \psi_{k}^{*}}{\mu \Omega_{s, s}}\right)^{m k}, \delta_{m}=\left(\frac{m \psi_{0}^{\prime}}{\mu \Omega_{s, s}}\right)^{m k}$, and $e_{n}=\sqrt{1-\phi_{n}^{2}}$. It is seen from (20) that a diversity order of $m k$ is achieved at $\mathrm{SR}_{k}$. An intuitive explanation is that if all the previous $(k-1)$ ordered channels fail, it declares an outage at the $k$ th user, which ensures a diversity order of $m k$.

3) Outage Throughput: Based on the analytical results for the outage probability of the secondary network, the outage throughput with the fixed transmission rates is

$$
\tau_{s}=\left(1-P_{1}\right) R_{1}+\cdots+\left(1-P_{K}\right) R_{K} .
$$

\begin{tabular}{|c|c|c|c|c|c|}
\hline \multicolumn{2}{|c|}{$\mathrm{PT} \longrightarrow \mathrm{ST}, \mathrm{PR}$} & \multicolumn{2}{|c|}{$\mathrm{ST} \longrightarrow \mathrm{PR}$} & \multicolumn{2}{|c|}{$\mathrm{ST} \longrightarrow \mathrm{SRs}$} \\
\hline \multicolumn{6}{|c|}{ (b) OMA-I scheme } \\
\hline $\mathrm{PT} \rightarrow \mathrm{ST}, \mathrm{PR}$ & & PR & $\mathrm{ST} \rightarrow \mathrm{SR}_{I}$ & ... & $\mathrm{ST} \rightarrow \mathrm{SR}_{k}$ \\
\hline
\end{tabular}

\section{OMA-Based Schemes for the Spectrum-Sharing CRN}

This subsection closes with a discussion of the OMA-based schemes for the considered spectrum-sharing CRN.

\begin{tabular}{|l|l|}
\hline $\mathrm{PT} \longrightarrow \mathrm{ST}, \mathrm{PR}, \mathrm{SRs}$ & $\mathrm{ST} \rightarrow \mathrm{PR}, \mathrm{SRs}$ \\
\hline
\end{tabular}

(a) NOMA-based scheme

(c) OMA-II and OMA-III schemes

Fig. 1. Transmission illustration of NOMA-based and OMA-based schemes.

1) OMA-I Scheme: Similar to the spectrum leasing scheme [6], a fraction of $1-\xi$ of the phase is used for the cooperative transmission of the primary user's information, and the remaining $\xi$ of the phase is dedicated to the secondary transmissions by employing NOMA, as shown in Fig. 1 (b). Therefore, the achievable data rate at PR can be expressed as

$$
R_{p}^{\mathrm{OMA}-\mathrm{I}}=\frac{1-\xi}{2} \log _{2}\left(1+\rho_{p}\left|f_{0}\right|^{2}+\rho_{s}\left|f_{2}\right|^{2}\right) .
$$

The achievable data rate at $\mathrm{SR}_{k}$ after $\mathrm{SIC}$ is obtained as

$$
R_{k}^{\mathrm{OMA}-\mathrm{I}}=\xi \log _{2}\left(1+\frac{\rho_{s}\left|h_{s, k}\right|^{2} \beta_{k}}{\rho_{s}\left|h_{s, k}\right|^{2} \sum_{i=k+1}^{K} \beta_{i}+1}\right)
$$

where $\beta_{i}, i \in \mathcal{K}$, denotes the power allocation coefficients.

2) OMA-II Scheme: By using conventional OMA (i.e. timedivision multiple access) for the secondary transmissions, the OMA-II scheme can be revised as follows. The first $1-\xi$ of the phase is same with the OMA-I scheme. The remaining $\xi$ of the phase is divided equally into $K$ time slots, as shown in Fig. 1 (c). The achievable data rate at $\mathrm{SR}_{k}$ during the $k$-th time slot is written as

$$
R_{k}^{\mathrm{OMA}-\mathrm{II}}=\frac{\xi}{K} \log _{2}\left(1+\rho_{s}\left|h_{s, k}\right|^{2}\right) .
$$

3) OMA-III Scheme: An opportunistic scheme is adopted in the remaining $\xi$ of the phase for the secondary access ${ }^{1}$, as depicted in Fig. 1 (c). The $k$ th user with the better channel conditions is allocated with a larger weight coefficient $\omega_{k}$, and the achievable data rate of the secondary network is

$$
R_{k}^{\mathrm{OMA}-\mathrm{III}}=\omega_{k} \xi \log _{2}\left(1+\rho_{s}\left|h_{s, k}\right|^{2}\right) .
$$

Moreover, the first $1-\xi$ of the phase is similar to the OMA-I and OMA-II schemes. Note that we can use (22)-(25) to derive the outage probability and the system throughput achieved by the OMA schemes. These results can be used as benchmarks to compare with our NOMA-based cooperative transmission scheme in the next section.

\footnotetext{
${ }^{1}$ This comes from exploiting the fluctuations of the fading channels, i.e. allocating more resources to the user with better channel conditions, instead of equally distributing the resources as in the OMA-II scheme.
} 


$$
\begin{aligned}
\tilde{P}_{k} \approx \tau_{k} \sum_{i=0}^{K-k} & \frac{(-1)^{i}}{k+i}\left(\begin{array}{c}
K-k \\
i
\end{array}\right)\left(\left(1-\exp \left(-\frac{m \psi_{k}^{*}}{\rho_{s} \Omega_{s, s}}\right) \sum_{l=0}^{m-1} \frac{\left(m \psi_{k}^{*} / \rho_{s} \Omega_{s, s}\right)^{l}}{l !}\right)^{k+i} \exp \left(-\frac{m \epsilon\left(\psi_{k}^{*}\right)}{\Omega_{p, s}}\right) \sum_{r=0}^{m-1} \frac{\left(m \epsilon\left(\psi_{k}^{*}\right) / \Omega_{p, s}\right)^{r}}{r !}\right. \\
& \left.+\frac{\epsilon\left(\psi_{k}^{*}\right) \pi}{2 N} \sum_{n=1}^{N} \sqrt{1-\phi_{n}^{2}}\left(1-\exp \left(-\frac{m \psi_{0}^{\prime}}{\rho_{s} \Omega_{s, s}}\right) \sum_{l=0}^{m-1} \frac{\left(m \psi_{0}^{\prime} / \rho_{s} \Omega_{s, s}\right)^{l}}{l !}\right)^{k+i} f_{\left|h_{p, k}\right|^{2}}\left(\frac{\left(\phi_{n}+1\right) \epsilon\left(\psi_{k}^{*}\right)}{2}\right)\right)
\end{aligned}
$$

\section{NUMERICAL EXAMPLES}

In this section, simulation results are presented to validate the efficiency of the NOMA-based cooperative transmission scheme. Without loss of generality, it is assumed that $\Omega_{f, 0}=$ $\Omega_{p, s}=0.1$ and $\Omega_{f, 1}=\Omega_{f, 2}=\Omega_{s, s}=0.2$. The power allocation coefficients are $\alpha_{0}=0.6, \alpha_{1}=0.3$ and $\alpha_{2}=0.1$. The Gaussian-Chebyshev parameter is chosen as $N=10$, since it generally provides a close approximation of the outage probability, and the phase allocation factor $\xi$ of the OMAbased schemes is set to $1 / 3$ for the illustration purpose ${ }^{2}$.

In Fig. 2, the outage performance of the primary network is shown as a function of the system SNR with different fading parameter $m$, where the target data rate is $R_{0}=0.5 \mathrm{bps} / \mathrm{Hz}$. It is observed that the proposed NOMA-based scheme achieves a lower outage probability than the OMA-based schemes for the primary network. The reason is that our proposed scheme can serve the primary and secondary users simultaneously without additional time/bandwidth, which effectively improves the spectrum utilization. It is also observed that all the curves (with the same the fading parameter $m$ ) have the same slope, due to the use of cooperative transmission for the primary network. Furthermore, it is worth pointing that the approximated results in (12) match perfectly the simulations, and the derived asymptotic results in (13) are very tight at high SNRs.

Fig. 3 provides the outage performance of the secondary network versus $\rho_{s}$. The target data rates are set the same as $R_{0}=R_{1}=R_{2}=0.5 \mathrm{bps} / \mathrm{Hz}$, and the power coefficients for OMA-I scheme are $\beta_{1}=0.8, \beta_{2}=0.2$. As expected, our proposed scheme outperforms the OMA-based schemes for most cases, and the diversity order at $\mathrm{SR}_{i}$ is a function of its ordered channels, which is consistent to (20). Interestingly, note that the OMA-I scheme achieves a lower outage probability than the proposed scheme for $\mathrm{SR}_{1}$. This can be explained by the fact that compared with the proposed scheme, more power is allocated to $\mathrm{SR}_{1}$ by the OMA-I scheme (i.e. $\beta_{1}=0.8$ for the OMA-I scheme and $\alpha_{1}=0.3$ for the proposed scheme), and the available transmit power dominates the outage probability in this case. This indicates the significance of the appropriate power allocation coefficients for NOMA.

Fig. 4 depicts the system throughput for the primary and secondary networks (i.e. $\tau_{p}+\tau_{s}$ ) based on different data rate settings. Clearly, our proposed scheme achieves the higher system throughput due to its lower outage probability for both networks. Note also that there exist throughput ceilings in the high-SNR regime, since the outage probability is approaching to zero and in this case, the system throughput is determined only by the target data rates of all users. Furthermore, the

\footnotetext{
${ }^{2}$ With an additional constraint, i.e. the minimum rate requirement of PR, the value of $\xi$ can be optimized for the OMA schemes. The consideration of such optimization is beyond the scope of this paper.
}

system throughput by the proposed scheme increases with an increase in the target data rates, as seen by the red and black lines. However, when $R_{0}=1 \mathrm{bps} / \mathrm{Hz}$, the system throughput of the proposed scheme is lowered and becomes worse than the OMA-based schemes. An intuitive explanation is that with the fixed power coefficients, the received SNR to detect $x_{0}$ cannot support the target threshold at $\mathrm{SR}_{i}$ with a larger $R_{0}$, and thus leads to the failure of the detection for $x_{1}$ and $x_{2}$. As a result, an outage will be declared at $\mathrm{SR}_{1}$ and $\mathrm{SR}_{2}$, which in return reduces the system throughput. Therefore, it is important to select the target data rates in designing NOMA schemes.

\section{CONCLUSION}

In this paper, a novel NOMA-based cooperative transmission scheme has been proposed for the spectrum-sharing CRN. The rationale behind is that the primary network shares the available spectrum with the secondary network in exchange for the cooperation, and thus the secondary transmitter is willing to transmit the primary message as well as its messages with employing NOMA signaling. This ensures that multiple users can be served simultaneously and the overall spectrum can be better utilized. Thus, an enhanced win-win situation for both networks can be achieved. Analytical expressions of the outage probability and the system throughput have been derived in closed forms. Substantial performance gains have been shown using the NOMA-based scheme than the conventional OMAbased schemes.

\section{Appendix A: Proof of TheOrem 1}

If $X$ is Nakagami- $m$ distributed with an integer fading parameter $m$, then its cumulative density function $F_{X}(x)$ can be expressed as [5]

$$
F_{X}(x)=1-\exp \left(-\frac{m x}{\Omega_{x}}\right) \sum_{l=0}^{m-1} \frac{\left(m x / \Omega_{x}\right)^{l}}{l !} .
$$

Then, we can rewrite $I_{1}$ in (11) as

$$
\begin{aligned}
I_{1} \stackrel{(\mathrm{a})}{=} \underbrace{\operatorname{Pr}\left(\left|f_{1}\right|^{2}>\frac{\psi_{0}}{\rho_{p}}\right)}_{I_{11}} \\
\quad \underbrace{\operatorname{Pr}\left(\rho_{p}\left|f_{0}\right|^{2}+\frac{\rho_{s}\left|f_{2}\right|^{2} \alpha_{0}}{\rho_{s}\left|f_{2}\right|^{2} \sum_{i=1}^{K} \alpha_{i}+1}<\psi_{0}\right)}_{I_{12}}
\end{aligned}
$$

where step (a) is due to the fact that all channels are independent. With the help of (26), $I_{11}$ is readily derived as

$$
I_{11}=\exp \left(-\frac{m \psi_{0}}{\rho_{p} \Omega_{f, 1}}\right) \sum_{s=0}^{m-1} \frac{\left(m \psi_{0} / \rho_{p} \Omega_{f, 1}\right)^{s}}{s !} .
$$




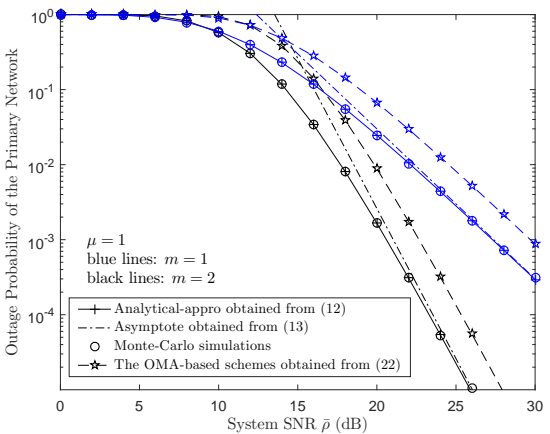

Fig. 2. Outage probability of the PR.

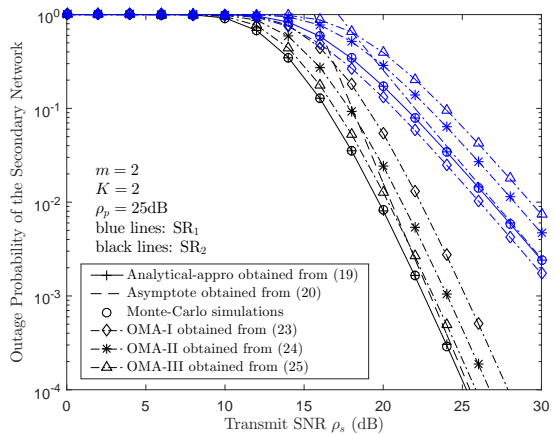

Fig. 3. Outage probability of the SRs.

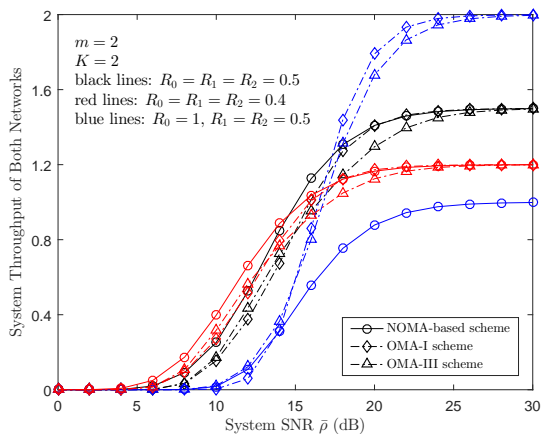

Fig. 4. System throughput of both networks.
Denote $Y=\frac{\rho_{s}\left|f_{2}\right|^{2} \alpha_{0}}{\rho_{s}\left|f_{2}\right|^{2} \sum_{i=1}^{K} \alpha_{i}+1}$, then $I_{12}$ can be calculated as

$$
\begin{aligned}
I_{12}= & \int_{0}^{\varpi} \operatorname{Pr}\left(\left|f_{0}\right|^{2}<\frac{\psi_{0}-y}{\rho_{p}}\right) f_{Y}(y) d y \\
=F_{Y}(\varpi) & -\int_{0}^{\varpi} \exp \left(-\frac{m\left(\psi_{0}-y\right)}{\rho_{p} \Omega_{f, 0}}\right) \\
& \times \sum_{r=0}^{m-1} \frac{\left(m\left(\psi_{0}-y\right) / \rho_{p} \Omega_{f, 0}\right)^{r}}{r !} f_{Y}(y) d y .
\end{aligned}
$$

However, it is challenging to obtain a closed-form expression for (29). In this case, by changing of variable $y=\frac{(x+1) \varpi}{2}$ and using Gaussian-Chebyshev quadrature [10, eq. (25.4.38)], we can approximate (29) as

$$
\begin{aligned}
I_{12} \approx F_{Y}(\varpi) & -\frac{\varpi \pi}{2 N} \sum_{n=1}^{N} \sum_{r=0}^{m-1} \sqrt{1-\theta_{n}^{2}} \\
& \times \exp \left(\frac{m\left(b_{n}-\psi_{0}\right)}{\rho_{p} \Omega_{f, 0}}\right) \frac{\left(m\left(\psi_{0}-b_{n}\right)\right)^{r}}{r !\left(\rho_{p} \Omega_{f, 0}\right)^{r}} f_{Y}\left(b_{n}\right)
\end{aligned}
$$

where $F_{Y}(\varpi)$ can be easily obtained by using (26). From (30), $f_{Y}(y)$ can be derived as

$$
\begin{aligned}
f_{Y}(y)= & \frac{m^{m}\left(y / \rho_{s}\left(\alpha_{0}-y \sum_{i=1}^{K} \alpha_{i}\right)\right)^{m-1}}{\Omega_{f, 2}^{m} \Gamma(m)} \\
& \times \exp \left(-\frac{m y}{\rho_{s} \Omega_{f, 2}\left(\alpha_{0}-y \sum_{i=1}^{K} \alpha_{i}\right)}\right)
\end{aligned}
$$

which is conditioned on $\alpha_{0}>y \sum_{i=1}^{K} \alpha_{i}$. By substituting the above results into (27), a closed-form for $I_{1}$ is obtained.

In addition, $I_{2}$ in (11) can be calculated as

$$
\begin{aligned}
I_{2}= & \operatorname{Pr}\left(\left|f_{1}\right|^{2}<\frac{\psi_{0}}{\rho_{p}}\right) \operatorname{Pr}\left(\left|f_{0}\right|^{2}<\frac{\psi_{0}}{\rho_{p}}\right) \\
= & \left(1-\exp \left(-\frac{m \psi_{0}}{\rho_{p} \Omega_{f, 1}}\right) \sum_{s=0}^{m-1} \frac{\left(m \psi_{0} / \rho_{p} \Omega_{f, 1}\right)^{s}}{s !}\right) \\
& \times\left(1-\exp \left(-\frac{m \psi_{0}}{\rho_{p} \Omega_{f, 0}}\right) \sum_{l=0}^{m-1} \frac{\left(m \psi_{0} / \rho_{p} \Omega_{f, 0}\right)^{l}}{l !}\right) .
\end{aligned}
$$

Now, combining $I_{1}$ and $I_{2}$ with (11), after some mathematical simplifications, Theorem 1 can be readily proved.

\section{ApPendix B: PRoOf OF TheOREM 2}

When $j=0$, we can rewrite (16) as

$$
\mathcal{O}_{k, 0}^{c} \triangleq\left\{\left|h_{s, k}\right|^{2}>\frac{\tilde{\psi}_{0}}{\rho_{s}}\right\}
$$

where $\tilde{\psi}_{0}=\frac{\psi_{0}-\rho_{p}\left|h_{p, k}\right|^{2}}{\alpha_{0}-\left(\psi_{0}-\rho_{p}\left|h_{p_{k}}\right|^{2}\right) \sum_{i=1}^{K} \alpha_{i}}$. It is assumed that $\alpha_{0}>\left(\psi_{0}-\rho_{p}\left|h_{p, k}\right|^{2}\right) \sum_{i=1}^{K} \alpha_{i}$, otherwise, an outage will be declared at $\mathrm{SR}_{k}$.

When $1 \leq j \leq k$, (17) can be rewritten as

$$
\mathcal{O}_{k, j}^{c} \triangleq\left\{\left|h_{s, k}\right|^{2}>\frac{\tilde{\psi}_{j}}{\rho_{s}}\right\}
$$

where $\tilde{\psi}_{j}=\frac{\psi_{j}}{\alpha_{j}-\psi_{j} \sum_{i=j+1}^{K} \alpha_{i}}$, by assuming a necessary condition as $\alpha_{j}>\psi_{j} \sum_{i=j+1}^{K} \alpha_{i}$. Denote $\psi_{k}^{*}=\max \left\{\tilde{\psi}_{1}, \cdots, \tilde{\psi}_{k}\right\}$, the outage probability in (15) can be reformulated as

$$
\begin{aligned}
\tilde{P}_{\text {out }, k}= & 1-\operatorname{Pr}\left(\left|h_{s, k}\right|^{2}>\frac{\max \left\{\tilde{\psi}_{0}, \psi_{k}^{*}\right\}}{\rho_{s}}\right) \\
= & \underbrace{\int_{\epsilon\left(\psi_{k}^{*}\right)}^{\infty} \operatorname{Pr}\left(\left|h_{s, k}\right|^{2}<\frac{\psi_{k}^{*}}{\rho_{s}}\right) f_{\left|h_{p, k}\right|^{2}}(x) d x}_{I_{3}} \\
& +\underbrace{\int_{0}^{\epsilon\left(\psi_{k}^{*}\right)} \operatorname{Pr}\left(\left|h_{s, k}\right|^{2}<\frac{\tilde{\psi}_{0}}{\rho_{s}}\right) f_{\left|h_{p, k}\right|^{2}}(x) d x}_{I_{4}} .
\end{aligned}
$$

By using binomial expansion and order statistics [11], $I_{3}$ can be further calculated as

$$
\begin{array}{r}
I_{3}=\tau_{k} \sum_{i=0}^{K-k} \frac{(-1)^{i}}{k+i}\left(\begin{array}{c}
K-k \\
i
\end{array}\right)\left(F_{\left|\tilde{h}_{s, k}\right|^{2}}\left(\frac{\psi_{k}^{*}}{\rho_{s}}\right)\right)^{k+i} \\
\times\left(1-F_{\left|h_{p, k}\right|^{2}}\left(\epsilon\left(\psi_{k}^{*}\right)\right)\right)
\end{array}
$$

where $\left|\tilde{h}_{s, k}\right|^{2}$ denotes the unordered channel gain. However, it is challenging to attain an exact closed form for $I_{4}$. In the following, the Gaussian-Chebyshev quadrature [10, eq. (25.4.38)] is used to find an approximation, shown by

$$
\begin{aligned}
I_{4} \approx & \frac{\epsilon\left(\psi_{k}^{*}\right) \pi \tau_{k}}{2 N} \sum_{n=1}^{N} \sum_{i=0}^{K-k} \frac{(-1)^{i}}{k+i}\left(\begin{array}{c}
K-k \\
i
\end{array}\right) \sqrt{1-\phi_{n}^{2}} \\
& \times\left(F_{\left|\tilde{h}_{s, k}\right|^{2}}\left(\frac{\tilde{\psi}_{0}^{\prime}}{\rho_{s}}\right)\right)^{k+i} f_{\left|h_{p, k}\right|^{2}}\left(\frac{\left(\phi_{n}+1\right) \epsilon\left(\psi_{k}^{*}\right)}{2}\right)
\end{aligned}
$$

in which $f_{\left|h_{p, k}\right|^{2}}(z)$ is derived readily as

$$
f_{\left|h_{p, k}\right|^{2}}(z)=\frac{m^{m} z^{m-1}}{\Omega_{p, s} \Gamma(m)} \exp \left(-\frac{m z}{\Omega_{p, s}}\right) .
$$

Hence, combining the foregoing results with (35), one can prove Theorem 2 straightforwardly. 


\section{REFERENCES}

[1] Z. Ding, Z. Yang, P. Fan, and H. V. Poor, "On the performance of non-orthogonal multiple access in $5 \mathrm{G}$ systems with randomly deployed users," IEEE Signal Process. Lett., vol. 21, no. 12, pp. 1501-1505, Dec. 2014.

[2] M. Al-Imari, P. Xiao, M. Imran, and R. Tafazolli, "Uplink nonorthogonal multiple access for 5G wireless networks," in Proc. 11th Int. Symp. Wireless Commun. Syst. (ISWCS), Aug. 2014, pp. 781-785.

[3] S. Timotheou and I. Krikidis, "Fairness for non-orthogonal multiple access in 5G systems," IEEE Signal Process. Lett., vol. 22, no. 10, pp. 1647-1651, Oct. 2015.

[4] Z. Ding, M. Peng, and H. V. Poor, "Cooperative non-orthogonal multiple access in 5G systems," IEEE Commun. Lett., vol. 19, no. 8, pp. 14621465, Aug. 2015

[5] J. Men, J. Ge, and C. Zhang, "Performance analysis of non-orthogonal multiple access for relaying networks over Nakagami- $m$ fading channels," IEEE Trans. Veh. Technol., to be published.
[6] O. Simeone, I. Stanojev, S. Savazzi, Y. Bar-Ness, U. Spagnolini, and R. Pickholtz, "Spectrum leasing to cooperating secondary ad hoc networks," IEEE J. Sel. Areas Commun., vol. 26, no. 1, pp. 203-213, Jan. 2008.

[7] R. Fan, Y. Zheng, J. An, H. Jiang, and X. Li, "Dynamic pricing over multiple rounds of spectrum leasing in cognitive radio," IEEE Trans. Veh. Technol., vol. 65, no. 3, pp. 1782-1789, Mar. 2016.

[8] Y. Liu, Z Ding, M. Elkashlan, and J. Yuan, "Non-orthogonal multiple access in large-scale underlay cognitive radio networks," IEEE Trans. Veh. Technol., to be published.

[9] L. Lv, J. Chen, and Q. Ni, "Cooperative non-orthogonal multiple access in cognitive radio," IEEE Commun. Lett., vol. 20, no. 10, pp. 2059-2062, Oct. 2016.

[10] M. Abramowitz and I. Stegun, Handbook of Mathematical Functions with Formulas, Graphs, and Mathematical Tables, New York, NY, USA: Dover, 1972.

[11] H. A. David and N. Nagaraja, Order Statistics, 3rd ed. John Wiley \& Sons, Inc., 2003. 